\title{
Preintervention MCP-1 serum levels as an early predictive marker of tumor response in patients with hepatocellular carcinoma undergoing transarterial chemoembolization
}

\author{
Ran You, Hao Jiang, Qingyu Xu, Guowen Yin \\ Department of Interventional Radiology, Jiangsu Cancer Hospital \& Jiangsu Institute of Cancer Research \& The Affiliated Cancer Hospital of \\ Nanjing Medical University, Nanjing, China \\ Contributions: (I) Conception and design: R You, Q Xu; (II) Administrative support: Q Xu, G Yin; (III) Provision of study materials or patients: \\ Q Xu, G Yin; (IV) Collection and assembly of data: R You, Hao Jiang, G Yin; (V) Data analysis and interpretation: R You; (VI) Manuscript writing: \\ All authors; (VII) Final approval of manuscript: All authors. \\ Correspondence to: Qingyu Xu, MD; Guowen Yin, MD. Department of Interventional Radiology, Jiangsu Cancer Hospital \& Jiangsu Institute of \\ Cancer Research \& The Affiliated Cancer Hospital of Nanjing Medical University, Nanjing, China. \\ Email: xqy1984king@163.com; jsnjygw@163.com.
}

Background: Transarterial chemoembolization (TACE) is a widely accepted treatment for unresectable or intermediate-stage hepatocellular carcinoma (HCC). However, response rates to TACE are heterogeneous and it is not fully understood which patients benefit most from TACE therapy in terms of tumor response. To identify the possible predictive roles of the perioperative monocyte chemoattractant protein-1 (MCP-1) levels in patients of HCC treated with TACE.

Methods: Forty patients of HCC receiving TACE were enrolled in a single center prospective observational study. MCP-1 and miR-210 levels were measured in 40 HCC patients at baseline before TACE and compared with 17 healthy controls by immunoassay and reverse transcriptase quantitative polymerase chain reaction (RT-qPCR). Tumor response assessments were taken after TACE treatment 4-6 weeks. Univariate and multivariate analysis were conducted to analyze factors correlated with tumor response in a Logistic regression model. The predictive roles of the involved variables on tumor response in patients with HCC suffering TACE were examined by receiver operating characteristic (ROC) curve analysis.

Results: The serum MCP-1 and miR-210 levels were significantly elevated in HCC patients compared to healthy subjects. Patients with the low preintervention MCP-1 and miR-210 levels attained a higher probability of achieving an objective response (OR) (88.5\% vs.42.9\%, $\mathrm{P}=0.007 ; 76.9 \%$ vs. 35.7\%, $\mathrm{P}=0.010$, respectively). Pre-TACE MCP-1 level $(<816.63 \mathrm{pg} / \mathrm{mL})$ was an independent risk factor associated with OR after TACE by univariate and multivariate analysis while Pre-TACE miR-210 level $(<4.43$ relative expression) was just positive by univariate analysis. ROC curve analysis showed that a combined index based on those two factors exhibited optimal predictive power of tumor response among all the involved variables (area under the curve $=0.823,95 \%$ CI: 0.681-0.965). Additionally, high pre-TACE serum MCP-1 level was correlated with cirrhosis, vascular invasion and Barcelona Clinic Liver Cancer (BCLC) stage. Elevated preTACE serum miR-210 level was associated with and BCLC stage.

Conclusions: The study demonstrates that the pre-TACE serum MCP-1 level serves as an effective predictor for tumor response. These findings probably help discriminate HCC patients pre-TACE who specially benefit from TACE regarding OR.

Keywords: Monocyte chemoattractant protein-1 (MCP-1); miR-210; hepatocellular carcinoma (HCC); transarterial chemoembolization (TACE) 
Submitted Aug 26, 2020. Accepted for publication Dec 04, 2020.

doi: $10.21037 /$ tcr-20-2791

View this article at: http://dx.doi.org/10.21037/tcr-20-2791

\section{Introduction}

Transarterial chemoembolization (TACE) is the most frequently applied local-regional treatment, and an approved first choice therapy for the management of BCLC B hepatocellular carcinoma (HCC) (1-3). Surgical resection is regarded as the only radical treatment of HCC. However, the 5-year recurrence rate after hepatectomy reaches approximately $70 \%$ (3). Therefore, investigation to improve the survival of HCC patients after radical resection is essential. TACE is a treatment modality resulting from tumor ischemic necrosis due to arterial embolization. TACE has shown improved survival rates in patients after radical resection (3). However not all HCC patients will benefit from TACE in terms of tumor response (4). Thus, how to choose patients who may benefit from TACE treatment appears urgent. To achieve the goal, great efforts have been made in recent years $(5,6)$. For patients with huge tumor size, dispersion distribution or impaired liver function, TACE may not be the first choice treatment. For patients who do not benefit from TACE, early identification of serum markers seems particularly important for they can be conveniently and noninvasively examined at different time period compared with tumor biopsy or imaging-based metrics.

Monocyte chemoattractant protein-1 (MCP-1; CCL2) is a member of the $\mathrm{C}-\mathrm{C}$ chemokine family which has been shown to be a therapeutic target for the treatment of injury and infection disease (7). MCP-1 also plays important roles in tumorgenesis, metastasis, recurrence, angiogenesis, epithelial-mesenchymal transition (EMT), biomarker and drug tolerance of HCC. Kim et al. found that Yapdependent MCP-1 expression could facilitate the normal liver growth by prohibiting macrophage infiltration in hepatocytes by Hippo signal path (8). MCP-1 can activate Hh and TGF- $\beta$ pathways in HCC cells to promote metastasis $(9,10)$. MCP-1 was an independent predictor for postoperative recurrence of HCC and overall survival (11). High level of MCP-1 will help angiogenesis and metastasis of HCC by FGFR3 signal path (12). Hedgehog signaling path promotes HCC invasion and EMT in vitro by MCP1/CCR2 axis (13). Moreover, former investigations have demonstrated that MCP-1 level is an encouraging tumor biomarker in HCC (14). MCP-1 induced tumor-associated neutrophils recruit macrophages and T-Regulatory cells to promote resistance to Sorafenib (15). However, few studies focused on MCP-1 levels in HCC patients receiving TACE treatment which is pensive of inflammation and the immune reaction for its hypoxic tumor microenvironment.

MicroRNAs (miRNAs) are a family of evolutionarily conserved non-coding RNAs. MicroRNA-210 (miR-210) is recognized as the major miRNA induced by hypoxia, involved in multiple processes in the hypoxia pathway. For HCC, it plays an important role in cancer angiogenesis, metastasis $(16,17)$. Zhan et al. performed a retrospective study and found that after TACE miR-210 changed at different time points closely associated with tumor progression (18).

Thus, we carried out a prospective observational study and sought to testify whether pre-TACE MCP-1 and miR210 serum levels can act as a predictor of tumor response in patients of HCC suffering TACE. We present the following article in accordance with the MDAR checklist (available at http://dx. doi. org/10.21037/tcr-20-2791).

\section{Methods}

\section{Patients and study design}

The prospective observation study was conducted in Jiang Su Cancer Hospital from October 2018 to July 2019. This study was registered in the Chinese Clinical Trial Register (registration number ChiCTR-1800017781). The purpose of the study was to determine the role of preintervention MCP-1 and miR-210 serum levels in tumor response of HCC patients received TACE therapy. HCC diagnosis was conducted with the guideline of the European Association for the Study of the Liver (19). Patients enrolled should meet criteria as the following: (I) TACE was the initial treatment; (II) Eastern Cooperative Oncology Group Performance Status (ECOG PS) of 0-1; (III) no serious infection before TACE; (IV) Child-Pugh classification A or B. Exclusion criteria: (I) Failed to get informed consent; (II) Multiple cancers; (III) Lack of histological diagnosis; (IV) No follow-up information. A total of 40 HCC patients were involved in the investigation. Sample size calculation was 
Table 1 Baseline characteristics of the hepatocellular carcinoma of study population

\begin{tabular}{lc}
\hline Characteristics & $\mathrm{HCC}(\mathrm{n}=40)$ \\
\hline Sex, male/female & $37 / 3$ \\
Age, mean $\pm \mathrm{SD}$, years & $59.4 \pm 10.6$ \\
Liver cirrhosis, absence/presence & $19 / 21$ \\
Maximum tumor size, mean $\pm \mathrm{SD}, \mathrm{cm}$ & $6.8 \pm 3.9$ \\
Vascular invasion, absence/presence & $26 / 14$ \\
AST, median (range), U/L & $42.5(8.0-227.0)$ \\
ALT, median (range), U/L & $36.0(17.0-153.0)$ \\
ALB, median (range), g/dL & $41.5(29.1-49.5)$ \\
TBIL, median (range), $\mu \mathrm{mol} / \mathrm{L}$ & $13.0(5.1-138.3)$ \\
AFP, median (range), $\mu \mathrm{gg} / \mathrm{mL}$ & $49.2(1.4-14,966.0)$ \\
Child-Pugh classification, A/B & $38 / 2$ \\
BCLC stage, B/C & $24 / 16$ \\
ECOG PS, 0/1 & $14 / 26$ \\
HBsAg, absence/presence & $3 / 37$ \\
\hline
\end{tabular}

HCC, hepatocellular carcinoma; AST, aspartate aminotransferase; ALT, alanine aminotransferase; ALB, albumin; TBIL, total bilirubin; AFP, alpha-fetoprotein; BCLC, Barcelona Clinic Liver Cancer; ECOG PS, Eastern Cooperative Oncology Group Performance Status; HBsAg, hepatitis B surface antigen.

based on the preliminary experiment using PASS Software (NCSS, LLC, USA). In the pre-experiment, MCP-1 was measured from 13 HCC patients. The ORR rate was $51.61 \%$. MCP-1 achieve a sensitivity of $80 \%$ at a specificity of $87.5 \%$. The sample size was chosen in order to ensure a power of 0.8 , with a type I error threshold of 0.05 . The minimum sample size of 20 patients was calculated based on sensitivity, while at least 33 patients were calculated based on specificity. Therefore, a total of 40 patients were selected for the study. Patient demographic was presented in Table 1. The study was conducted in accordance with the Declaration of Helsinki (as revised in 2013). The protocol for this study and the use of patient blood samples were approved by the ethics committee of the JiangSu Cancer Hospital (2018-050). All patients agreed to participate and signed a written consent.

\section{Measurement of serum MCP-1 concentration}

HCC patient serum samples were collected before TACE, while for the control group, were collected form 17 healthy volunteers, and then separated the serum by centrifugation at 3,000 rpm for 10 minutes within 2 hours. Samples were stored at -80 degrees for further examination. Serum MCP1 concentration was measured by ELISA using the Human monocyte chemotactic protein 1 ELISA kit (ab179886, abcam, USA). Absorption at $450 \mathrm{~nm}$ was measured using a microplate reader (Bio-Rad, USA). A standard curve was set up for ELISA (using a four-parameter logistic curve fit). The absorbance values were transferred into the software GraphPadPrism and samples concentrations were back-calculated using the standard curve fitted with four parameters. The detection range for serum MCP-1 ELISA was 4.7 to $300 \mathrm{pg} / \mathrm{mL}$ with a sensitivity of $1.26 \mathrm{pg} / \mathrm{mL}$ and inter-assay variabilities of $<10 \%$. Serum samples were diluted 10-fold in PBS and each dilution was then subjected to ELISA assay. Three biological repeats were performed, in triplicate. The linear regression $\left(\mathrm{r}^{2}\right)$ of our curve was closely linear with an average of $r^{2}>0.97$.

\section{Quantification of miR-210 expression levels}

Total RNA was extracted from serum using Trizol (Invitrogen, USA) following the manufacturer's protocol. Reverse transcriptase quantitative polymerase chain reaction (RT-qPCR) was performed on the extracted RNA from the serum samples. The primers for miR-210 were 5 '-ACACTCCAGCTGGGCTGTGCGTGTGAC AGCGG-3' and 5'-CTCAACTGGTGTCGTGGA-3'. The real time RT-PCR was performed according to the following reaction condition: $10 \mathrm{~min}$ at $95^{\circ} \mathrm{C}$ followed by 50 cycles of $15 \mathrm{~s}$ at $95^{\circ} \mathrm{C}$ and $1 \mathrm{~min}$ at $60^{\circ} \mathrm{C}$. miR-210 relative expression was examined using the delta-delta cycle threshold value $\left(2^{-\Delta \Delta C t}\right)$ method relative to the $\mathrm{U} 6$ as the endogenous control. RT-PCR analysis was performed on a [RT-PCR machine]. All PCR reactions were replicated three times.

\section{TACE and evaluation of tumor response}

All enrolled patients underwent imaging with a contrastenhanced CT or MR imaging protocol before and after 4-6 weeks of the initial TACE. TACE procedures were performed by two interventional radiologist (with over 10 years of experience in HCC interventions) followed by standardized approach. Tumor response was evaluated by three experienced radiologists according to the modified Response Evaluation Criteria in Solid Tumors (mRECIST). OR means patients with objective response (OR), including 
complete or partial remission (CR + PR). Non-OR (nonresponding), represents patients in the condition of stable or progressive disease (SD + PD).

\section{Statistical analyses}

Statistical analysis was performed by SPSS version 25.0 and GraphPad Prism version 8.0 software. Differences between groups were determined by non-parametric Mann-Whitney $\mathrm{U}$ test or $t$-test for continuous variables. Chi-square tests or Fisher's exact test were used for categorical data. Receiver operating characteristic (ROC) was constructed to identify the ability of the potential factors for discriminating HCC patients or predicting tumor response. The cut-off values were assessed according to the highest Youden index. Logistic regression was applied to analyze the multivariate variables with tumor response. Statistical significance was considered as $\mathrm{P}<0.05$.

\section{Results}

\section{Baseline characteristics of the HCC study population.}

In our study, we included 40 HCC patients treated with TACE. The baseline characteristics of the HCC study population were shown in Table 1. The mean age of the patients was 59.4 years. Nearly $92.5 \%$ were men. The mean maximum tumor size was $6.8 \mathrm{~cm}$. Among the patients, about half $(19 / 40,47.5 \%)$ patients with liver cirrhosis. Most patients $(38 / 40,95 \%)$ were in Child-Pugh classification A with good liver function. Based on the Barcelona Clinic Liver Cancer (BCLC) classification, $60 \%$ and $40 \%$ of the patients had the stage of $B$ and $C$, respectively. $87.5 \%$ of the enrolled patients had a good ECOG PS (0-1).

\section{Serum levels of MCP-1 and miR-210 were elevated in HCC patients with TACE compared to bealthy controls.}

The serum concentration of MCP-1 by ELISA assay was significantly elevated in patients with HCC patients compared to healthy controls (Figure 1A). At the same time, RTqPCR and analysis via the $2^{-\Delta \Delta C t}$ method revealed increased expression levels of miR-210 in the HCC patients when compared with healthy controls (Figure 1B). Mean MCP1 concentrations for the HCC patients were higher relative to healthy controls (617.62 vs. $131.47 \mathrm{pg} / \mathrm{mL}$, Figure $1 A$, Table S1), while mean miR-210 levels for the HCC patients was 4.62 relative to healthy controls (Figure $1 B$,
Table S1). ROC analyses suggest that serum MCP-1 and miR-210 expression levels distinguish HCC patients from healthy controls with an area under the curve of 0.918 (95\% CI: 0.848-0.988) and 0.872 (95\% CI: 0.772-0.972), respectively (Figure 1C,D).

\section{Preintervention serum MCP-1 and miR-210 levels predict tumor response after TACE}

Our data showed that serum levels of MCP-1 and miR210 can distinguish HCC patients from healthy controls. And then, we further explored the predictive value of preinterventional serum levels for the response to TACE therapy. According to the modified RECIST criteria (20), we divided our patients into two groups. One group is OR, means patients with OR, including complete or partial remission after TACE for 4-6 weeks. The control group is non-OR (non-responding), represents patients in the condition of stable or progressive disease after TACE for 4-6 weeks. Our data shows that the OR patients after TACE has the lower level of preintervention serum MCP-1 and miR-210 compared to the Non-OR patients (Figure $2 A, B$ ). Consistent with this, the AUC values of MCP-1 and miR-210 serum levels can distinguish between OR and non-OR patients by ROC curve analysis $(0.739$, 0.698 , respectively; Figure 2C). The 95\% confidence interval for MCP-1 and miR-210 distinguish OR from non-OR patients were (0.574-0.904) and (0.516-0.880) respectively. At the threshold of $816.63 \mathrm{pg} / \mathrm{mL}, \mathrm{MCP}-1$ sensitivity was $57.1 \%$ and specificity was $88.5 \%$, whereas miR-210 sensitivity was $64.3 \%$ and specificity was $76.9 \%$ at the threshold of 4.43 (Table S2). As shown in Table S3, patients with low preintervention MCP-1 and miR-210 serum levels have more opportunity to have OR after TACE $(88.5 \%$ vs. $42.9 \%, \mathrm{P}=0.007 ; 76.9 \%$ vs. $35.7 \%, \mathrm{P}=0.010$, respectively). Based on our results, we took the preintervention MCP1 and miR-210 serum levels for the combined diagnostic factor. The formula followed on the two variables logistic regression analysis. Combined preintervention MCP-1 and miR-210 $=-2.068+2.273^{*}$ (if preintervention MCP-1 serum levels $>816.63 \mathrm{pg} / \mathrm{mL}$ ) $+1.734^{*}$ (if preintervention miR-210 serum levels $>4.43$ relative expression) (Table S4). Combined the preintervention MCP-1 and miR-210 serum levels can significant improve the sensitivity and little decrease the specificity $(85.7 \%, 68.2 \%$, respectively, Table S2). Then, we explored which can predict the best value of tumor response in HCC Patients undergoing TACE. ROC curves indicated that combined the preintervention MCP- 
A

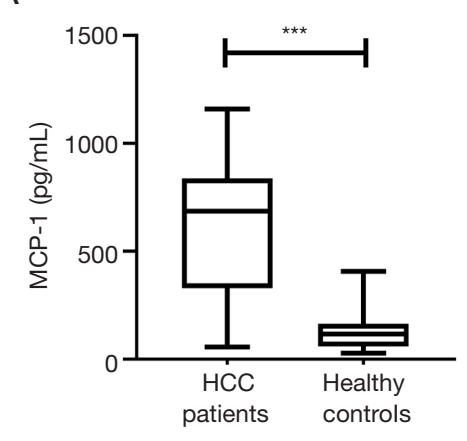

B

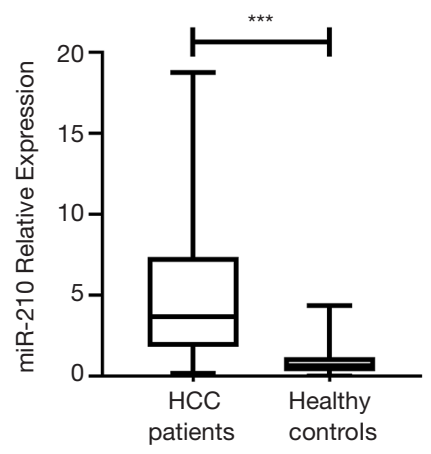

C

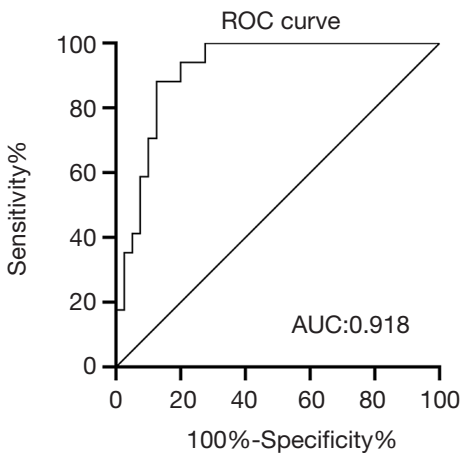

D

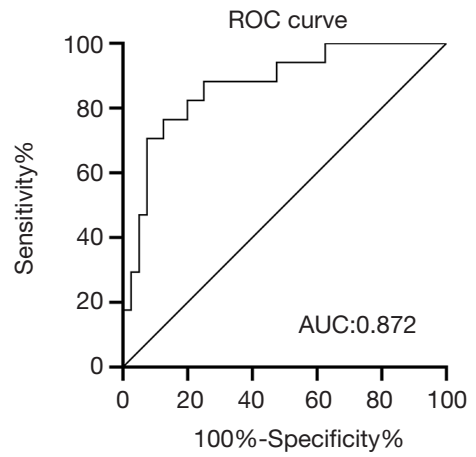

Figure 1 Serum levels of MCP-1 and miR-210 were elevated in HCC patients with TACE compared to healthy controls. (A) Mean expression levels of serum MCP-1 was determined by ELISA analysis; (B) miR-210 were determined by RT-qPCR and analyzed using the delta-delta cycle threshold value $(2-\Delta \Delta \mathrm{Ct}$ ) method relative to the healthy controls. The midline in box plots represents the median, and the upper and lower limits depicting the maximum and minimum values, respectively. (C,D) ROC curves were estimated for MCP-1 and miR210.AUC indicated 0.918 and 0.872 for MCP-1 and miR-210 regarding the discrimination between HCC patients with TACE and healthy controls. $\mathrm{N}=40$ and 17 for TACE patients and healthy controls, respectively. ${ }^{* * *}, \mathrm{P}<0.001$. MCP-1, monocyte chemoattractant protein-1; TACE, transarterial chemoembolization; HCC, hepatocellular carcinoma; miR, microRNA; ROC, receiver operating characteristic; AUC, area under the curve; HCC, hepatocellular carcinoma.

1 and miR-210 serum levels had the best prediction value of tumor response (AUC $=0.823 ; 95 \%$ CI: 0.681-0.965) than MCP-1 (AUC $=0.739$ 95\% CI: 0.574-0.904) or miR210 (AUC $=0.698 ; 95 \%$ CI: 0.516-0.880) alone. Other indicators including AFP (AUC $=0.563 ; 95 \%$ CI: 0.390 0.770), BCLC stage (AUC $=0.522 ; 95 \%$ CI: $0.332-0.712$ ), and Child-Pugh classification (AUC $=0.571 ; 95 \% \mathrm{CI}$ : $0.377-0.766$; Table 2). To investigate the potential predictors of TACE therapy, chi-square test was used to assess the parameters. We observed that liver cirrhosis $(\mathrm{P}=0.015)$, the preintervention MCP-1 serum levels $(\mathrm{P}=0.007)$, and the preintervention miR-210 serum levels $(\mathrm{P}=0.010)$ were significantly associated with OR (Table 3). And then, for multivariate analysis, we found the preintervention MCP1 serum levels was an independent risk factors for OR ( $\mathrm{P}=0.035$; 95\% CI: 1.143-43.275; Table 4). We did not conclude the scoring systems of Child-Pugh classification or BCLC stage mainly avoiding the interference of collinearity factors. These scoring systems can be drawn from the variables such as vascular invasion and serum albumin etc.

\section{Associations of preintervention MCP-1 and miR-210 serum levels in patients baseline characteristics}

To further explore the associations between serum levels and patients' baseline characteristics, we carried out the chi-square test to examine the relationship. The results presented that preintervention MCP-1 level correlated with vascular invasion $(\mathrm{P}=0.007)$, liver cirrhosis $(\mathrm{P}=0.002)$ and BCLC stage $(\mathrm{P}=0.025$, Table 5). Meanwhile, miR-210 level was found to correlate with vascular invasion $(\mathrm{P}=0.010)$ and BCLC stage ( $\mathrm{P}=0.008$, Table 6). 
A

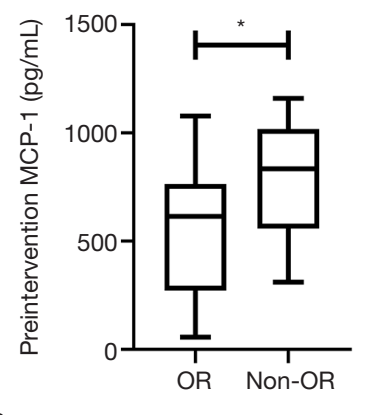

B

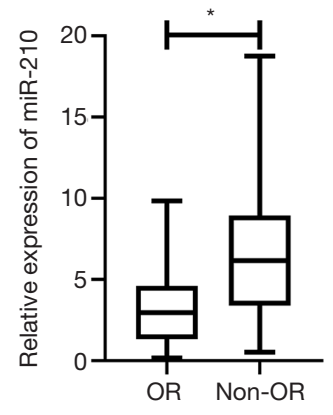

C

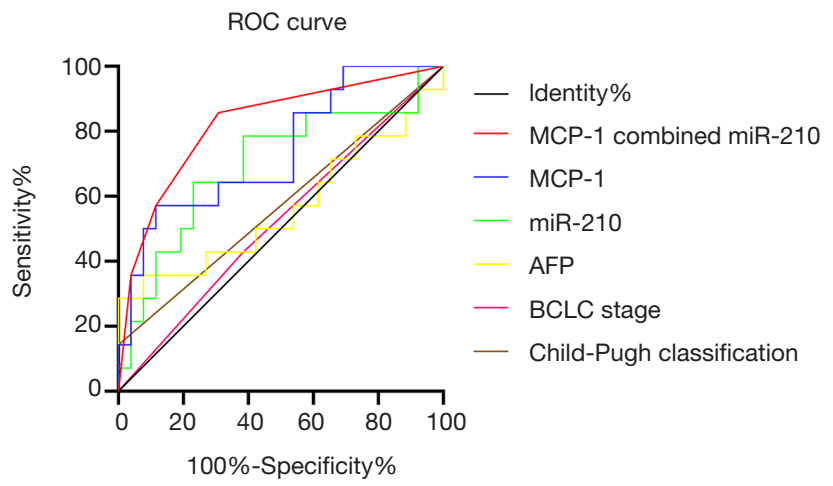

Figure 2 Preintervention MCP-1 and miR-210 serum levels predict tumor response after TACE. (A,B) Compared to the Non-OR patients, OR patients has lower preintervention serum MCP-1 and miR-210 levels. The midline in box plots represents the median, and the upper and lower limits depicting the maximum and minimum values, respectively. (C) ROC curves analysis distinguish the OR and Non-OR patients. N=26 and 14 for OR and non-OR to HCC patients treated with TACE, respectively. ${ }^{*}, \mathrm{P}<0.05$. MCP-1, monocyte chemoattractant protein-1; TACE, transarterial chemoembolization; Non-OR, non-responding; miR, microRNA; OR, objective response; ROC, receiver operating characteristic; HCC, hepatocellular carcinoma.

Table 2 Discriminant abilities of the variables examined

\begin{tabular}{llll}
\hline Variables & AUC & P value & OR (95\% Cl) \\
\hline AFP & 0.580 & 0.411 & $0.390-0.770$ \\
BCLC stage & 0.522 & 0.821 & $0.332-0.712$ \\
Child-Pugh classification & 0.571 & 0.461 & $0.377-0.766$ \\
MCP-1 & 0.739 & 0.014 & $0.574-0.904$ \\
miR-210 & 0.698 & 0.041 & $0.516-0.880$ \\
MCP-1 combined miR-210 & 0.823 & 0.001 & $0.681-0.965$ \\
\hline
\end{tabular}

AUC, area under the curve; OR, objective response; BCLC, Barcelona Clinic Liver Cancer; MCP-1, monocyte chemoattractant protein-1.

\section{Discussion}

Increasing evidence indicates a key role of MCP-1 in the process of carcinogenesis, progression, metastasis in HCC. This study is the first to demonstrate the predictive role of the preintervention MCP-1 level in tumor response of patients with HCC undergoing TACE. We found that serum MCP-1 level in HCC patient increased significantly contrast with healthy individuals which is in accordance with Wang's study (14). Pre-TACE MCP-1 level (<816.63 pg/mL) was an independent risk factor associated with OR after 
Table 3 Characteristics Associated tumor response with OR and non-OR group

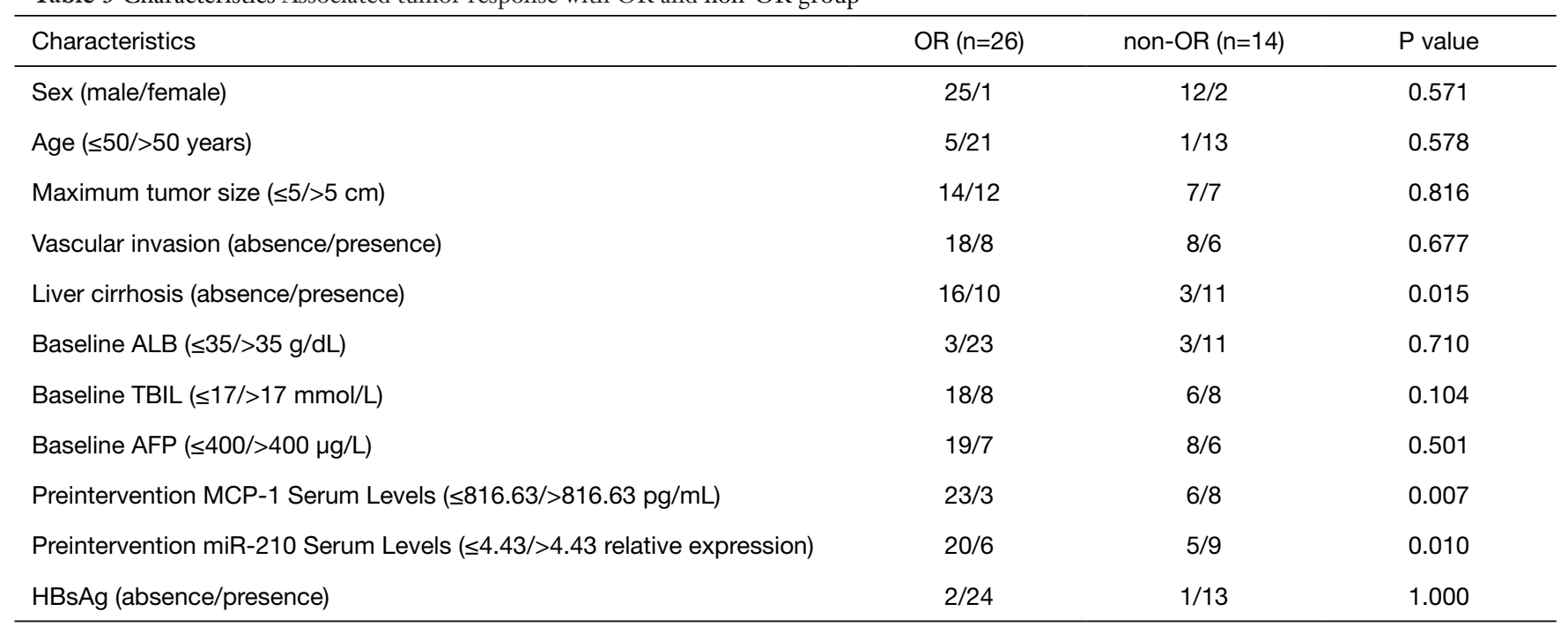

OR, objective response; ALB, albumin; TBIL, total bilirubin; AFP, alpha-fetoprotein; MCP-1, monocyte chemoattractant protein-1; miR, microRNA; HBsAg, hepatitis B surface antigen.

Table 4 Multivariate Logistic regression analyses for tumor response

\begin{tabular}{lcc}
\hline \multirow{2}{*}{ Variables } & \multicolumn{2}{c}{ Multivariate analysis } \\
\cline { 2 - 3 } & OR $(95 \% \mathrm{Cl})$ & $\mathrm{P}$ value \\
\hline AFP & $0.295-9.838$ & 0.551 \\
Liver cirrhosis & $0.579-19.970$ & 0.176 \\
MCP-1 & $1.143-43.275$ & 0.035 \\
miR-210 & $0.854-25.120$ & 0.075 \\
\hline
\end{tabular}

OR, objective response; AFP, alpha-fetoprotein; MCP-1, monocyte chemoattractant protein-1; miR, microRNA.

TACE. The pre-TACE MCP-1 level seems to be an effective predictor of OR in HCC. We hold the option that pre-TACE MCP-1 level (>816.63 pg/mL) may represent immune and inflammatory response before treatment, after chemoembolization hypoxia tumor microenvironment will aggravate immune and inflammatory response which is a poor prognosis for HCC patients. CC chemokines, especially MCP-1 is major attractant of monocyte and macrophage precursor to the tumor microenvironment. Thus, MCP1 can be regarded as biomarkers of inflammation. After TACE, hypoxia elicits inflammation response. It has documented that the systemic inflammation response index can predicting prognosis in HCC patients. It is a better predictor of OS than AFP and Child stage. It appeared superior to clinical Child stage and AFP level in prognosis prediction (21).
One explanation probably lies in inflammatory factors play a crucial role in promoting proliferation, invasion and metastasis of malignant cells. MCP-1 plays important roles in tumorgenesis, metastasis, recurrence, angiogenesis, EMT, biomarker and drug tolerance of HCC. In addition, tumor-resident MSCs expressed high levels of CCL2/ MCP-1 promoting tumor growth by recruiting monocytes/ macrophages (22). Functionally, experimental studies have demonstrated that MCP-1 induced by VEGF participate in angiogenesis, promoting macrophage recruitment, collagen deposition, and tumor recurrence (23). Moreover, persistent activation of the MCP-1/CCR2 signaling pathway is detrimental to the patient, which may result in EMT metastasis $(13,24)$. Highly expressed MCP-1 level can inhibit cellular immunity by helping $\mathrm{T}$ cells differentiate into TH2 cells but not TH1 cells (25). MiR-210 known as the major miRNA induced by hypoxia also increased evidently because of HCC hypoxia microenvironment (26). Distinctly elevated miR-210 was proved to be another significant predictor for OR in our study. Specially, a new criterion based on the preTACE MCP-1 level was found to display the best predictive role among all the variables measured. Nevertheless, MCP-1 combined with miR-210 presented better predictive power of tumor response.

Meanwhile, the data demonstrated that elevated preTACE plasma MCP-1 level was correlated with vascular invasion, cirrhosis, and BCLC stage. The explanation may be that the elevating MCP-1 level was closely related to HCC 
Table 5 Associations between preintervention MCP-1 serum levels and patients' characteristics

\begin{tabular}{|c|c|c|c|}
\hline Characteristics & \multicolumn{2}{|c|}{ Preintervention MCP-1 serum levels } & $P$ value \\
\hline Sex (male/female) & $27 / 2$ & $10 / 1$ & 1.000 \\
\hline Age $(\leq 50 />50$ years $)$ & $5 / 24$ & $1 / 10$ & 0.882 \\
\hline Maximum tumor size $(\leq 5 />5 \mathrm{~cm})$ & $17 / 12$ & $4 / 7$ & 0.208 \\
\hline Baseline ALB ( $\leq 35 />35 \mathrm{~g} / \mathrm{dL})$ & $4 / 25$ & $2 / 9$ & 1.000 \\
\hline Baseline TBIL ( $\leq 17 />17$ mmol/L) & $19 / 10$ & $5 / 6$ & 0.427 \\
\hline Baseline AFP $(\leq 400 />400 \mu \mathrm{g} / \mathrm{L})$ & $20 / 9$ & $7 / 4$ & 1.000 \\
\hline BCLC stage $(B / C)$ & $21 / 8$ & $3 / 8$ & 0.025 \\
\hline ECOG PS (0/1) & $8 / 21$ & $6 / 5$ & 0.221 \\
\hline HBsAg (absence/presence) & $2 / 27$ & $1 / 10$ & 1.000 \\
\hline
\end{tabular}

MCP-1, monocyte chemoattractant protein-1; ALB, albumin; TBIL, total bilirubin; AFP, alpha-fetoprotein; AST, aspartate aminotransferase; ALT, alanine aminotransferase; BCLC, Barcelona Clinic Liver Cancer; ECOG PS, Eastern Cooperative Oncology Group Performance Status; HBsAg, hepatitis B surface antigen.

Table 6 Associations between preintervention miR-210 serum levels and patients' characteristics

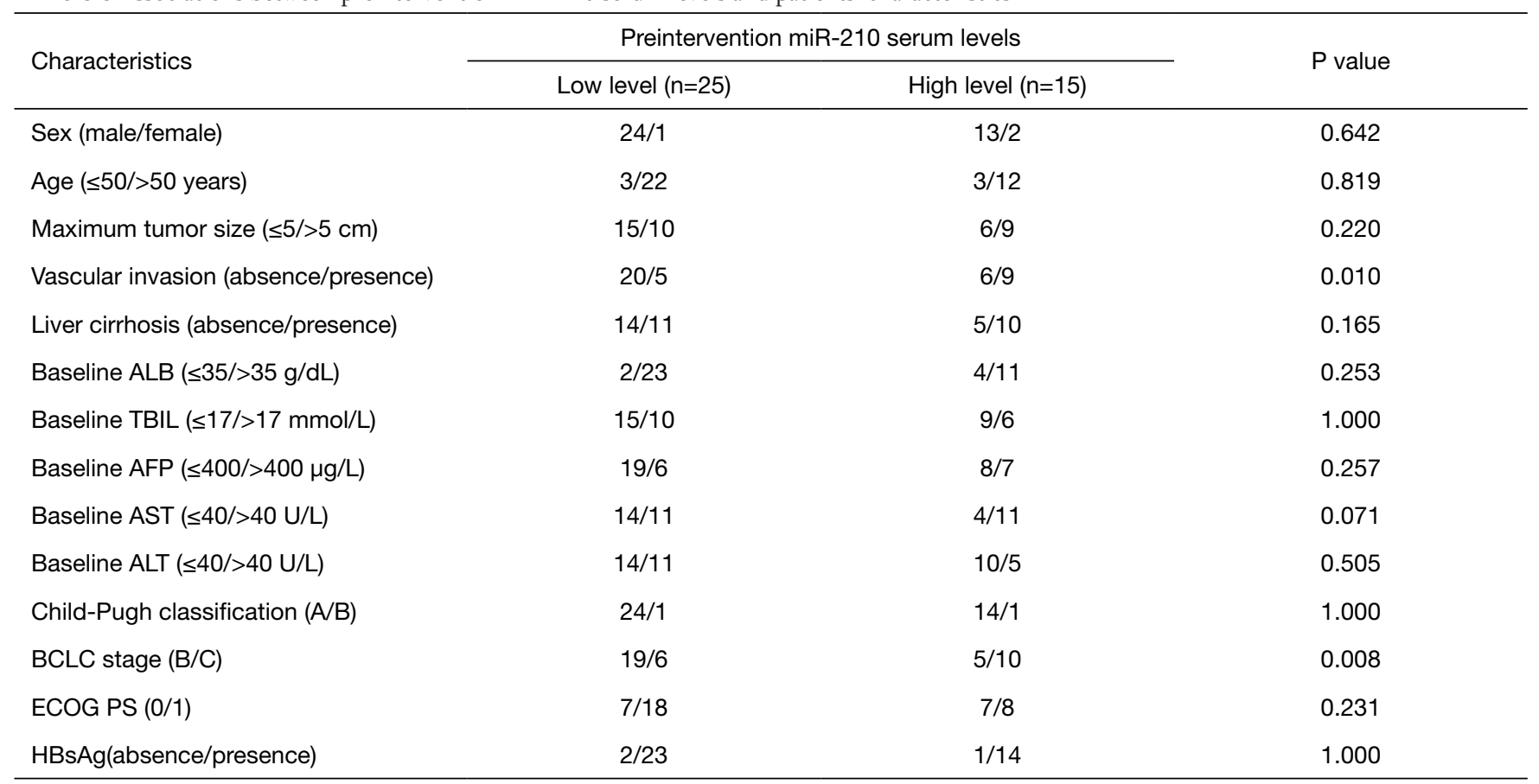

miR, microRNA; ALB, albumin; TBIL, total bilirubin; AFP, alpha-fetoprotein; AST, aspartate aminotransferase; ALT, alanine aminotransferase; BCLC, Barcelona Clinic Liver Cancer; ECOG PS, Eastern Cooperative Oncology Group Performance Status; HBsAg, hepatitis B surface antigen. 
progression and hepatic stellate cells up-regulates CCR2 by down-regulation of miR-19b-3p to promote liver fibrosis (27-29). High levels of miR-210 are reciprocally associated with BCLC stage which is in line with previous study (18).

The m-RECIST response is known to all that assessment of OR is crucial in the evaluation of the effect of anti-tumor treatment in HCC (30). The response rates after TACE diverge due to inadequately defined response criteria and various clinicopathologic features of patients. Obviously, the method can't anticipate the tumor response. Our study may provide an easily and noninvasively way to predict the tumor response.

The study has several limitations. Firstly, the study was carried out in a single center with a limited sample size. Secondly, only MCP-1 and miR-210 levels were detected in serum without HCC tissues. Thirdly, the relative level of miR-210 was measured but not the absolute concentration. The methods to examine the absolute concentration such as conjugated magnetic beads are needed (31). Finally, OS was not evaluated in the present study because of the limited follow-up.

In a word, the present investigation suggests that preTACE serum levels of MCP-1 acts as an early candidate marker for anticipating OR in TACE-treated patients with HCC. These findings may be useful in choosing patients who may benefit from TACE. Evidently, MCP-1 combined with miR-210 exhibited optimal predictive power of tumor response. A multi-center study with a huger number of patients and longer observation time is necessary for testing the results.

\section{Acknowledgments}

Funding: This work was supported by grants from National Natural Science Foundation of China (No. 81701803); the General Program of Jiangsu Commission of Health (Z2017004); the project of Natural Science Foundation of Jiangsu Cancer Hospital (ZM201802, 2017YQL-12); the Scientific Research Project of HengRui (HRIRF2018-C042); the young talents program of Jiangsu cancer hospital QL201806.

\section{Footnote}

Reporting Checklist: The authors have completed the MDAR checklist. Available at http://dx. doi.org/10.21037/ tcr-20-2791
Data Sharing Statement: Available at http://dx. doi. org/10.21037/tcr-20-2791

Conflicts of Interest: All authors have completed the ICMJE uniform disclosure form (available at http://dx. doi. org/10.21037/tcr-20-2791). The authors have no conflicts of interest to declare.

Ethical Statement: The authors are accountable for all aspects of the work in ensuring that questions related to the accuracy or integrity of any part of the work are appropriately investigated and resolved. The study was conducted in accordance with the Declaration of Helsinki (as revised in 2013), and approved by the ethics committee of the JiangSu Cancer Hospital (2018-050). All patients agreed to participate and signed a written consent. This study was registered in the Chinese Clinical Trial Register (registration number ChiCTR-1800017781).

Open Access Statement: This is an Open Access article distributed in accordance with the Creative Commons Attribution-NonCommercial-NoDerivs 4.0 International License (CC BY-NC-ND 4.0), which permits the noncommercial replication and distribution of the article with the strict proviso that no changes or edits are made and the original work is properly cited (including links to both the formal publication through the relevant DOI and the license). See: https://creativecommons.org/licenses/by-nc-nd/4.0/.

\section{References}

1. Sacco R, Tapete G, Simonetti N, et al. Transarterial chemoembolization for the treatment of hepatocellular carcinoma: a review. J Hepatocell Carcinoma 2017;4:105-10.

2. Forner A, Llovet JM, Bruix J. Hepatocellular carcinoma. Lancet 2012;379:1245-55.

3. Bruix J, Sherman M. Management of hepatocellular carcinoma: an update. Hepatology 2011;53:1020-2.

4. Finn RS, Zhu AX, Farah W, et al. Therapies for advanced stage hepatocellular carcinoma with macrovascular invasion or metastatic disease: A systematic review and meta-analysis. Hepatology 2018;67:422-35.

5. Sieghart $W$, Hucke F, Peck-Radosavljevic $M$. Transarterial Chemoembolization: Modalities, Indication, and Patient Selection. J Hepatol 2015;62:1187-95.

6. Galle PR, Tovoli F, Foerster F, et al. The treatment of 
intermediate stage tumours beyond TACE: From surgery to systemic therapy. J Hepatol 2017;67:173-83.

7. Deshmane SL, Kremlev S, Amini S, et al. Monocyte chemoattractant protein-1 (MCP-1): an overview. J Interferon Cytokine Res 2009;29:313-26.

8. Kim W, Khan SK, Liu Y, et al. Hepatic Hippo Signaling Inhibits Protumoural Microenvironment to Suppress Hepatocellular Carcinoma. Gut 2018;67:1692-703.

9. Shih YT, Wang MC, Zhou J, et al. Endothelial progenitors promote hepatocarcinoma intrahepatic metastasis through monocyte chemotactic protein-1 induction of microRNA-21. Gut 2015;64:1132-47.

10. Liu J, Chen S, Wang W, et al. Cancer-associated fibroblasts promote hepatocellular carcinoma metastasis through chemokine-activated hedgehog and TGF- $\beta$ pathways. Cancer Lett 2016;379:49-59.

11. Huang W, Chen Z, Zhang L, et al. Interleukin-8 Induces Expression of FOXC1 to Promote Transactivation of CXCR1 and CCL2 in Hepatocellular Carcinoma Cell Lines and Formation of Metastases in Mice. Gastroenterology 2015;149:1053-67.e14.

12. Liu X, Jing X, Cheng X, et al. FGFR3 promotes angiogenesis-dependent metastasis of hepatocellular carcinoma via facilitating MCP-1-mediated vascular formation. Med Oncol 2016;33:46.

13. Zhuang H, Cao G, Kou C, et al. CCL2/CCR2 axis induces hepatocellular carcinoma invasion and epithelial-mesenchymal transition invitro through activation of the Hedgehog pathway. Oncol Rep 2018;39:21-30.

14. Wang WW, Ang SF, Kumar R, et al. Identification of serum monocyte chemoattractant protein-1 and prolactin as potential tumor markers in hepatocellular carcinoma. PLoS One 2013;8:e68904.

15. Zhou SL, Zhou ZJ, Hu ZQ, et al. Tumor-Associated Neutrophils Recruit Macrophages and T-Regulatory Cells to Promote Progression of Hepatocellular Carcinoma and Resistance to Sorafenib. Gastroenterology 2016;150:1646-58.17.

16. Lin XJ, Fang JH, Yang XJ, et al. Hepatocellular Carcinoma Cell-Secreted Exosomal MicroRNA-210 Promotes Angiogenesis In Vitro and In Vivo. Mol Ther Nucleic Acids 2018;11:243-52.

17. Kai AK, Chan LK, Lo RC, et al. Down-regulation of TIMP 2 by HIF-1 $\alpha / \mathrm{miR}-210 / \mathrm{HIF}-3 \alpha$ regulatory feedback circuit enhances cancer metastasis in hepatocellular carcinoma. Hepatology 2016;64:473-87.
18. Zhan M, Li Y, Hu B, et al. Serum microRNA-210 as a predictive biomarker for treatment response and prognosis in patients with hepatocellular carcinoma undergoing transarterial chemoembolization. J Vasc Interv Radiol 2014;25:1279-87.e1.

19. Bruix J, Sherman M. Management of hepatocellular carcinoma. Hepatology 2005;42:1208-36.

20. Edeline J, Boucher E, Rolland Y, et al. Comparison of tumor response by Response Evaluation Criteria in Solid Tumors (RECIST) and modified RECIST in patients treated with sorafenib for hepatocellular carcinoma. Cancer 2012;118:147-56.

21. Xu L, Yu S, Zhuang L, et al. Systemic inflammation response index (SIRI) predicts prognosis in hepatocellular carcinoma patients. Oncotarget 2017;8:34954-60.

22. Ren G, Zhao X, Wang Y, et al. CCR2-dependent recruitment of macrophages by tumor-educated mesenchymal stromal cells promotes tumor development and is mimicked by TNF $\alpha$. Cell Stem Cell 2012;11:812-24.

23. Shantha Kumara HM, Myers EA, Herath SA, et al. Plasma monocyte chemotactic protein-1 remains elevated after minimally invasive colorectal cancer resection. World J Gastrointest Oncol 2014;6:413-9.

24. Jeong KY, Lee EJ, Kim SJ, et al. Irradiation-induced localization of IL-12-expressing mesenchymal stem cells to enhance the curative effect in murine metastatic hepatoma. Int J Cancer 2015;137:721-30.

25. Gu L, Tseng S, Horner RM, et al. Control of TH2 polarization by the chemokine monocyte chemoattractant protein-1. Nature 2000;404:407-11.

26. Mathew LK, Simon MC. mir-210: a sensor for hypoxic stress during tumorigenesis. Mol Cell 2009;35:737-8.

27. Duan M, Wang ZC, Wang XY, et al. TREM-1, an inflammatory modulator, is expressed in hepatocellular carcinoma cells and significantly promotes tumor progression. Ann Surg Oncol 2015;22:3121-9.

28. Wang D, Li X, Li J, et al. APOBEC3B interaction with PRC2 modulates microenvironment to promote HCC progression. Gut 2019;68:1846-57.

29. Lan T, Li C, Yang G, et al. Sphingosine kinase 1 promotes liver fibrosis by preventing miR-19b-3p-mediated inhibition of CCR2. Hepatology 2018;68:1070-86.

30. Jung ES, Kim JH, Yoon EL, et al. Comparison of the methods for tumor response assessment in patients with hepatocellular carcinoma undergoing transarterial 
chemoembolization. J Hepatol 2013;58:1181-7.

31. Hong CY, Chen X, Li J, et al. Direct detection of circulating microRNAs in serum of cancer patients by coupling protein-facilitated specific enrichment and rolling circle amplification. Chem Commun (Camb) 2014;50:3292-5.

Cite this article as: You $\mathrm{R}$, Jiang $\mathrm{H}, \mathrm{Xu} \mathrm{Q}$, Yin $\mathrm{G}$. Preintervention MCP-1 serum levels as an early predictive marker of tumor response in patients with hepatocellular carcinoma undergoing transarterial chemoembolization. Transl Cancer Res 2021;10(2):966-976. doi: 10.21037/tcr-20-2791 
Supplementary

Table S1 Serum levels of laboratory markers

\begin{tabular}{lcc}
\hline Variables & TACE patients mean (range) & Healthy controls mean (range) \\
\hline MCP-1 $(\mathrm{pg} / \mathrm{mL})$ & $617.62(57.35-1,158.87)$ & $131.47(27.57-406.27)$ \\
miR-210 (Relative expression) & $4.62(0.17-18.76)$ & $1.00(0.00-4.36)$ \\
\hline
\end{tabular}

TACE, transarterial chemoembolization; MCP-1, monocyte chemoattractant protein-1; miR, microRNA.

Table S2 Discriminant abilities of the variables examined

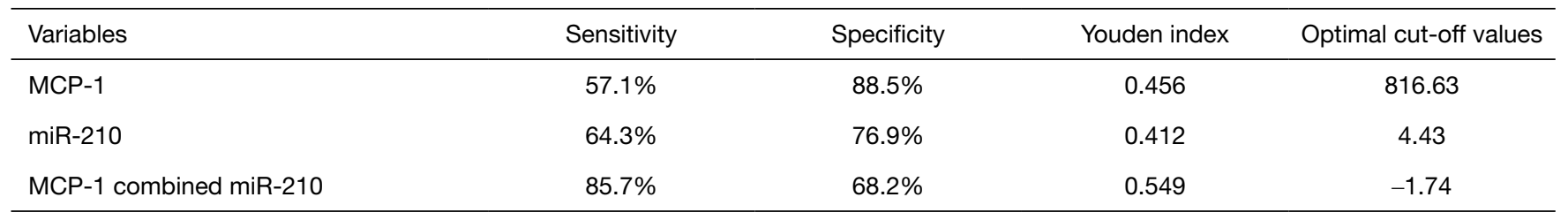

MCP-1, monocyte chemoattractant protein-1; miR, microRNA.

Table S3 Preintervention MCP-1 and miR-210 serum levels with objective response after TACE

\begin{tabular}{lcc}
\hline Characteristics & OR & non-OR \\
\hline Preintervention MCP-1 Serum Levels & & 0.007 \\
Low $(\leq 816.63 \mathrm{pg} / \mathrm{mL})$ & $23(88.5 \%)$ & $6(42.9 \%)$ \\
High $(>816.63 \mathrm{pg} / \mathrm{mL})$ & 3 & 8 \\
Preintervention miR-210 Serum Levels $(\leq 4.43 />4.43$ relative expression) & $20(76.9 \%)$ & $5(35.7 \%)$ \\
Low ( $\leq 4.43$ relative expression) & 6 & 9.010 \\
High $(>4.43$ relative expression) & 6 \\
\hline
\end{tabular}

MCP-1, monocyte chemoattractant protein-1; TACE, transarterial chemoembolization; OR, objective response; miR, microRNA.

Table S4 Multivariate logistic regression models of preintervention MCP-1 and miR-210 serum levels with tumor response

\begin{tabular}{lcccc}
\hline Variables & $\mathrm{B}$ & Wald & $\mathrm{P}$ value & $95 \% \mathrm{Cl}$ \\
\hline MCP-1 & 2.273 & 6.561 & 0.010 & $1.705-55.234$ \\
miR-210 & 1.734 & 4.429 & 0.035 & $1.126-28.457$ \\
Constant & -2.068 & 10.061 & & \\
\hline
\end{tabular}

MCP-1, monocyte chemoattractant protein-1; miR, microRNA. 